\title{
Chaos and anomalous diffusion of adatoms on solid surfaces
}

\author{
R. Guantes, J. L. Vega, and S. Miret-Artés \\ Instituto de Matemáticas y Física Fundamental, Consejo Superior de Investigaciones Científicas, Serrano, 123, 28006 Madrid, Spain
}

(Received 5 March 2001; revised manuscript received 1 June 2001; published 4 December 2001)

\begin{abstract}
The microscopic diffusional dynamics of $\mathrm{Na}$ adatoms on a $\mathrm{Cu}(001)$ surface is modeled by the classical deterministic motion of a particle from a two-dimensional periodic potential previously fitted to the experiment. Depending on the energy of the particle, a series of chaotic transitions take place determining transport properties of the system. Two different regimes of diffusion, anomalous and normal, are reported and related to the chaotic dynamics. Simple periodic orbits are responsible for frustrated vibrational motions as well as unusual high rate of migration paths along particular directions. The connection between Lévy flights and the principal periodic orbits of the system is established, as well as the validity of some statistical models proposed to describe the anomalous diffusion process. The influence of noise and dissipation on this dynamics is also briefly discussed.
\end{abstract}

DOI: 10.1103/PhysRevB.64.245415

\section{INTRODUCTION}

One of the most important elementary dynamical processes occurring on surfaces is the diffusional motion of atomic and molecular adsorbates. Diffusion of adsorbates is a preliminary step in more complicated surface phenomena such as, for example, associative desorption and heterogeneous catalysis, and strongly affects their overall efficiency. A detailed knowledge of the diffusion mechanisms, as well as the influence of the potential energy surface (PES) on the vibrational and diffusional dynamics of the adsorbates is an important question that needs increasing efforts both at experimental and theoretical level.

Present day technology allows for accurate experimental determination of diffusion coefficients as well as vibrational frequencies of adsorbates. Experimental techniques which can directly probe the microscopic dynamics, such as fieldion microscopy and scanning tunneling microscopy, have good atomic resolution but are restricted to relatively low diffusion mobilities $\left(D \sim 10^{-9} \mathrm{~cm}^{2} / \mathrm{s}\right)$. Recently, the technique of quasielastic helium-atom scattering $(\mathrm{QHAS})^{1}$ has emerged as a valuable and alternative tool for studying the dynamics of adsorbates. This method could be considered as the counterpart surface technique of the well-known method of quasielastic neutron scattering ${ }^{2}$ used to study diffusion in the bulk, and allows one to follow the atomic motion continuously on an atomic length- and time-scale by probing the diffusing species with a beam of helium atoms. In analogy with the theory of liquids, the ensemble of diffusing classical particles is described by a time-dependent pair-correlation function $^{3}$ whose Fourier transform, the dynamic structure factor, is directly provided by the QHAS experiment leading, therefore, to a complete information on the dynamical behavior of the system. Additional dynamical aspects can also be extracted from such measurements in order to discriminate diffusion mechanisms and establish the role of long jumps in the diffusion process.

The diffusion of $\mathrm{Na}$ atoms at low coverages on a $\mathrm{Cu}(001)$ surface has been recent and extensively investigated experimentally with the QHAS technique, as well as theoretically within the Langevin and Fokker-Planck formalisms. ${ }^{4,5} \mathrm{We}$ think that this system could be considered as a good proto-
PACS number(s): 68.43.-h, 79.20.Rf, 05.40.Fb, 05.45.-a

type for further developments and investigations of the theory. At low coverage of $\mathrm{Na}$ atoms, the adsorbateadsorbate interactions can be disregarded and the corresponding experimental features have been interpreted only in terms of the adsorbate-substrate interaction governed by a nonseparable PES, whose parameters were fitted to experimental results using molecular dynamics simulations. ${ }^{5}$ In the first theoretical treatments of this system, ${ }^{4,6}$ a simple separable cosine potential was chosen for the PES which could not explain all the experimental findings, in particular, the marked anisotropy in diffusion along different directions.

On the other hand, the study of transport properties of Hamiltonian systems is being the subject of increasing interest and fast developments. ${ }^{7,8}$ It has been widely demonstrated that a deterministic chaotic dynamics can mimic the dynamics of a rich variety of stochastic processes. Among many other important questions, the connection between the phase space structure of a Hamiltonian system and the observation of anomalous diffusion processes and Lévy flights is one of the most interesting topics. ${ }^{9}$ Anomalous diffusion is characterized by mean squared displacements diverging faster than linearly in time and it is a generic phenomenon appearing in conservative as well as dissipative systems. Processes occurring in fluid dynamics and semiconductor microstructures are some examples where anomalous diffusion has been shown to play an important role. ${ }^{9,10}$ The term Lévy flight is used to indicate a random walk in a continuous $n$-dimensional space displaying a stable or Lévy distribution of jump lengths and an average finite time between jumps. Opposite to the Brownian motion, which relies on the central limit theorem establishing a Gaussian distribution as the limiting distribution of the sum of a very high number of independent and identically distributed random variables, the socalled Weierstrass random walk is characterized by limiting stable or Lévy distributions, ${ }^{11}$ whose distinguishing property is the presence of long-range power law tails which may lead to a divergence of even the lowest-order moments.

In this paper our aim is threefold. First, to carry out a detailed investigation of the classical regular and chaotic dynamics of the $\mathrm{Na}$ particles (neglecting friction and thermal effects) on the $\mathrm{Cu}(001)$ surface to establish some basic microscopic mechanisms by which deterministic diffusion pro- 
ceeds. Second, to reconsider the importance of long jumps and the high rate of diffusion paths along the direction diagonal to the saddle point barrier, as suggested by the experiments, in the light of the classical dynamics. And third, following studies of deterministic diffusion in other periodic potentials ${ }^{12-14}$ we will show that, in the $\mathrm{Na} / \mathrm{Cu}(001)$ potential, anomalously enhanced diffusion can take place due to the existence of long jumps influenced by a hierarchy of stable periodic orbits associated to diffusive motions of the adparticles. Depending on the energy regime considered, the stabilities of some simple periodic orbits originate a competition between long free motions which promote the diffusion and trapped vibrational motions inside the potential wells, and this makes the adparticles behave in a similar manner to a Weierstrass random walk.

The paper is organized as follows. In the next section, we describe very briefly the basic theory relating the diffusion coefficient to observables such as the dynamic structure factor and the power spectrum, as well as the model employed in the calculations. In Sec. III, a detailed analysis of the classical dynamics of the system as we change the energy is carried out. The calculation of mean squared displacements and velocity power spectra shows the existence of distinct diffusion mechanisms giving rise to anomalous and normal diffusion. Some statistical models proposed to explain the anomalous diffusive dynamics in terms of Lévy flights are also tested. Finally, in Sec. IV, a physical discussion relating our findings to the actual knowledge of this system, where a complete study has been carried out in Ref. 5, and some conclusions are reported.

\section{THEORY AND MODEL POTENTIAL}

\section{A. Theoretical formulation for atom-surface diffusion}

The quantity measured in QHAS experiments is the differential reflection probability (the probability of a projectile helium atom to be scattered from the diffusing collective into a certain solid angle $\Omega$ with an energy exchange $\hbar \omega$ ) which is given by the following expression: ${ }^{1}$

$$
\frac{d^{2} R(\Delta \mathbf{K}, \omega)}{d \Omega d \omega}=n_{d} F^{2} \iint G(\mathbf{R}, t) e^{i(\Delta \mathbf{K} \cdot \mathbf{R}-\omega t)} d \mathbf{R} d t,
$$

where $\Delta \mathbf{K}$ is the wave vector transfer parallel to the surface, $n_{d}$ the diffusing particle concentration on the surface, and $F$ the atomic form factor depending on the interaction potential between the $\mathrm{He}$ atoms and the adparticles. The double integral in the right part of Eq. (1) is the dynamic structure factor $S(\Delta \mathbf{K}, \omega)$. The dynamical information is contained in the time-dependent pair correlation function $G(\mathbf{R}, t)$ which is the ensemble averaged probability density of finding an atom at the position $\mathbf{R}$ at time $t$, given that an atom was at the origin at some arbitrary time $t=0$. At low adatom concentrations, when interactions between adsorbates can be neglected, the $G$ function is only described by the so-called "self-part," that is, the dynamics for a single diffusing particle $G(\mathbf{R}, t)$ $\equiv G_{s}(\mathbf{R}, t)$. Then $G_{s}(\mathbf{R}, t)$ obeys a Fokker-Planck equation for one particle moving in a periodic potential under the influence of temperature and friction (a Klein-Kramers equation ${ }^{15}$ ) or, equivalently, the dynamics of the individual atoms is also given by a Langevin equation of the type

$$
m \ddot{\mathbf{R}}=-\nabla V(\mathbf{R})-\eta \dot{\mathbf{R}}+\mathbf{F}_{\mathbf{r}}(t)
$$

with $m$ the mass of the adparticles, $\eta$ the friction coefficient, $\mathbf{F}_{\mathbf{r}}(t)$ a random fluctuating force with zero mean and deltacorrelated in time and $V(\mathbf{R})$ the adiabatic adsorption potential. A procedure employed to obtain such a potential has consisted in starting with a model potential containing some adjustable parameters, and solving the above Langevin equation for different friction coefficients to reproduce the experimental QHAS measurements. ${ }^{5}$ For this end the dynamic structure factor is better expressed as

$$
\begin{aligned}
S_{s}(\Delta \mathbf{K}, \omega) & =\int d t e^{-i \omega t}\left\langle e^{-i \Delta \mathbf{K} \cdot \mathbf{R}(t)} e^{i \Delta \mathbf{K} \cdot \mathbf{R}(0)}\right\rangle \\
& =\int d t e^{-i \omega t} I_{s}(\Delta \mathbf{K}, t),
\end{aligned}
$$

where the brackets in the integral denote an ensemble average. The intermediate scattering function for the self-part $I_{s}(\Delta \mathbf{K}, t)$ is just the spatial Fourier transform of $G_{s}(\mathbf{R}, t)$. Scattering at small $\Delta \mathbf{K}$ provides information on long distance correlations and $\mathrm{He}$ atoms are mainly sensitive to the macroscopic continuous diffusional motion of the adatoms. Assuming a random continuous motion on a flat surface, corresponding to a Brownian dynamics, the time-dependent pair correlation function $G_{s}(\mathbf{R}, t)$ is a Gaussian distribution (the solution of a diffusion equation) for which the dynamic structure factor gives a Lorentzian function

$$
S_{s}(\Delta \mathbf{K}, \omega)=\frac{1}{\pi} \frac{D \Delta \mathbf{K}^{2}}{\omega^{2}+D^{2} \Delta \mathbf{K}^{4}}
$$

and the diffusion coefficient $D$ can be extracted from the full width at half maximum (FWHM) $\Gamma=2 D\left|\Delta \mathbf{K}^{2}\right|$. It is worth noting that strictly speaking the dynamic structure factor comes from the solution of a Klein-Kramers equation. This has been numerically solved for one-dimensional and twodimensional periodic potentials ${ }^{6,16}$ and, under some approximations, the dynamic structure factor can still be considered as Lorentzian but the FWHM has a more complicated functional form than the $\Delta \mathbf{K}^{2}$ dependence. Assuming a jump diffusion model, ${ }^{17}$ this functional form can be related to the total jump rate and jump probabilities. This model is valid whenever the diffusion is a thermally activated process. In the low friction and low temperature regimes, the Langevin equation (2) reduces to Newton's classical equations of motion, and the diffussion becomes a process controlled by Hamiltonian dynamics, which will give rise to different diffusion mechanisms as explained in the next section.

Another quantity directly related to the diffusion coefficient is the velocity power spectrum, or Fourier transform of the velocity autocorrelation function defined as

$$
Z(\omega)=\int_{-\infty}^{\infty}\langle\mathbf{v}(t) \cdot \mathbf{v}(0)\rangle e^{-i \omega t} d t .
$$

The diffusion coefficient is simply (in two dimensions) 


$$
D=\frac{1}{2} Z(\omega=0)
$$

When diffusion is normal, the velocity power spectrum should converge to a finite value as $\omega$ tends to zero. On the other hand, a divergence of the power spectrum like $\omega^{-\alpha}$ with $0<\alpha \leqslant 1$ is the fingerprint of anomalous diffusion. ${ }^{9}$ Anomalous diffusion can also be identified from different quantities such as the mean squared displacement (MSD) which asymptotically grows faster than linearly in time $\left\langle\mathbf{R}^{2}(t)\right\rangle \sim t^{1+\alpha}$. The velocity power spectrum is convenient since it can be simply related to the self dynamic structure factor. It has been shown ${ }^{3}$ that the velocity autocorrelation function and the intermediate "self-scattering" function fulfill the following expression:

$$
\langle\mathbf{v}(t) \cdot \mathbf{v}(0)\rangle=-\lim _{\Delta \mathbf{K} \rightarrow 0} \frac{1}{\Delta \mathbf{K}^{2}} \frac{d^{2} I_{s}(\Delta \mathbf{K}, t)}{d t^{2}}
$$

and by Fourier transforming both sides we obtain the relation

$$
Z(\omega)=\omega^{2} \lim _{\Delta \mathbf{K} \rightarrow 0} \frac{S_{s}(\Delta \mathbf{K}, \omega)}{\Delta \mathbf{K}^{2}} .
$$

Note that, for normal diffusion, the limit $\Delta \mathbf{K} \rightarrow 0$ of the ratio $S_{s}(\Delta \mathbf{K}, \omega) / \Delta \mathbf{K}^{2}$ should behave as $D / \omega^{2}$ for small $\omega$ according to Eq. (6). The Lorentzian function in Eq. (4) gives the expected limit $D / \omega^{2}$. For anomalous diffusion, Eqs. (4) and (8) above should be generalized taking into account that $G_{s}(\mathbf{R}, t)$ is not a Gaussian distribution, as explained in Sec. III B.

\section{B. Potential energy surface}

The systematic or adiabatic force representing the interaction of the $\mathrm{Na}$ atoms with the $\mathrm{Cu}$ surface is derived from a semiempirical potential as given in Ref. 5, whose main features we describe here. Since the vibrations of the $\mathrm{Na}$ adatoms normal to the surface have a much higher frequency ${ }^{18}$ than those parallel to the surface (the frustrated translational mode or $T$ mode), the perpendicular coordinate $z$ can be fixed and the PES has been considered as being averaged over the normal vibrations. Therefore the potential is a two-dimensional function of only the in-plane coordinates $x$ and $y$.

From theoretical calculations of the dynamic structure factor at different $\Delta \mathbf{K}$ and temperatures as measured by the QHAS technique, ${ }^{5}$ a periodic potential has been fitted to the form

$$
V(x, y)=V_{0}(x, y)+V_{1}(x, y)+V_{2}(x, y) .
$$

The first term is a simple separable cosine potential

$$
V_{0}(x, y)=V_{0}[2-\cos (2 \pi x / a)-\cos (2 \pi y / a)],
$$

where $a$ is the lattice constant of the $\mathrm{Cu}(001)$ surface ( $a$ $=2.557 \AA$ ), and $V_{0}=41.4 \mathrm{meV}$. The second term is added to produce a lowering of the potential barrier at on-top sites according to the observations

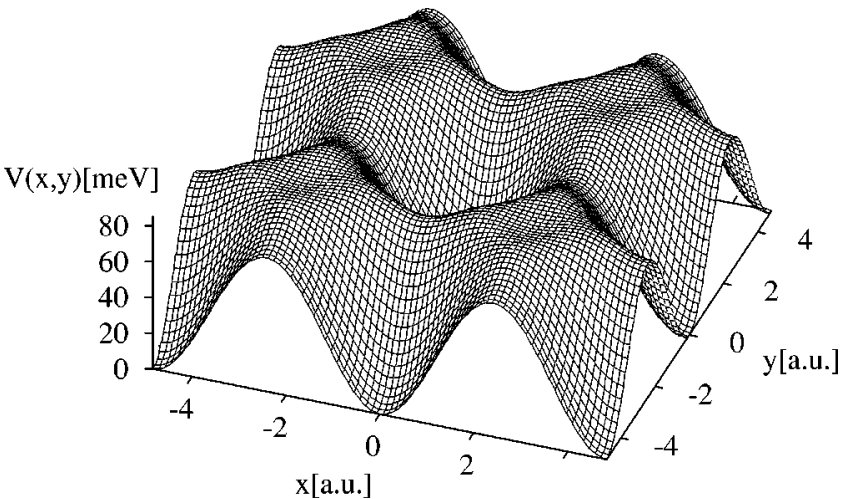

FIG. 1. Two-dimensional semiempirical potential. The zero of the energy axis is taken at the energy of the hollow sites minima $(x=y=0, \pm a, \ldots)$. The $x, y$ directions correspond to the $[1 \overline{1} 0]$ and [110] azimuths, respectively, and the barrier for motion along this direction is $74.64 \mathrm{meV}$. The diagonal directions have Miller indices [100] and [010] and the energy barrier is at $84.49 \mathrm{meV}$.

$$
\begin{aligned}
V_{1}(x, y)= & -A \sum_{m, n} \exp \left(-b\left\{\left[x / a-\left(m+\frac{1}{2}\right)\right]^{2}\right.\right. \\
& \left.\left.+\left[y / a-\left(n+\frac{1}{2}\right)\right]^{2}\right\}\right)
\end{aligned}
$$

with $A=2 V_{0}$ and $b=11.8$. Finally, the third term is a nonseparable part which serves to alter the curvature near the minima and vary the difference between the potential at the minima and the bridge positions

$$
\begin{aligned}
V_{2}(x, y)= & C V_{0} \pi^{2} \sum_{m, n}\left[(x / a-n)^{2}+(y / a-m)^{2}\right] \\
& \times \exp \left[-(x / a-m)^{2}-(y / a-n)^{2}\right]
\end{aligned}
$$

with $C=-0.2$. Note that for a periodic potential, the sums in Eqs. (11) and (12) must run over the entire set of integer pairs $(m, n)$. In practice, for the classical trajectory simulations, we reduce the dynamics to a single Wigner-Seitz cell by imposing periodic boundary conditions and the sum over Gaussians is truncated up to some few terms (typically $m$ and $n$ vary between -10 and 10). In Fig. 1 we show a 3D plot of the corresponding PES. The $x$ and $y$ directions are taken along the azimuths with Miller indices [110] and [110], respectively. The energy zero is taken at the minima of the potential wells corresponding to hollow sites. The saddle point barrier along the $x$ or $y$ directions is at $74.64 \mathrm{meV}$, and the saddle point barrier along the diagonal [100] or [010] azimuths is at $84.49 \mathrm{meV}$. The small minima on the potential truncated hills correspond to on-top sites above the copper atoms and are at energy $82.74 \mathrm{meV}$. As will be shown later, they will be responsible for the anomalously high rate of migration paths along the diagonal [100] direction detected in the experiment. ${ }^{5}$ The maxima at the top hills are located at $85.51 \mathrm{meV}$.

An important feature of the present PES is its nonseparability, as opposed to a previous PES used to reproduce the experiments. ${ }^{4}$ The nonseparability of the adiabatic potential 
can influence considerably the occurrence of long jumps and the dependence of the diffusion coefficient with friction. ${ }^{19}$

\section{DETERMINISTIC DIFFUSION}

\section{A. Chaotic dynamics}

Here we analyze in detail the classical dynamics of the system at different energies. Below the saddle-point barrier, at $E_{s}=74.64 \mathrm{meV}$, we have only localized, intra-well motions. Between this energy value and the maximum at on-top sites $E_{\max }=85.51 \mathrm{meV}, \mathrm{Na}$ atoms diffuse along the $x$ or $y$ directions with a predominance of long paths interrupted by episodes where the particle is trapped for a while inside a well. For $E>E_{\max }$, the particle can in principle diffuse freely although we will also see that coexistence of long jumps with trapped motions persist for some ranges of energy. With increasing energies, $E \gg E_{\max }$, an integrable (free particle) problem is approached. This will happen to be the case for energies greater than $200 \mathrm{meV}$. Indeed, what is observed for energies not too high above $E_{\max }$ is a more irregular dynamics than those for energies closer to the saddle-point barrier.

The study of the phase space structure begins usually with the analysis of the Poincare surface of section, which is a mapping of the phase space obtained by means of keeping fixed one of the dynamical variables at a constant value. The Poincaré surface of section gives a qualitative idea of the degree of irregularity at a given energy. To obtain a more complete view of the dynamics at any energy, one can locate the most relevant periodic orbits of the system and follow their evolution (changes of stability and bifurcations) with energy. ${ }^{20}$ The main families of periodic motion usually emerge from the equilibrium points of the potential. ${ }^{21}$ As we vary the energy, a periodic orbit can bifurcate (generate new periodic motions) and change its stability. The stabilities of the periodic motions are important since they determine the behavior of the nearby classical trajectories, thus dividing the phase space into regular and chaotic regions. It is well known that the dynamics of hyperbolic chaotic systems (all periodic orbits are unstable) is equivalent to a usual random walk dynamics (Brownian motion) ${ }^{7}$ and then diffusion is expected to be normal. Coexistence of regular and chaotic dynamics can give rise to different diffusion regimes, including anomalous diffusion, and the completely regular or integrable situation is expected to produce ballistic diffusion (the MSD grows asymptotically as $t^{2}$ ) since the trajectories follow almost unperturbed straight paths.

The stability and possible bifurcations of a periodic orbit can be found by calculating the trace of the so-called stability or monodromy matrix $\mathbf{M}$ (the Jacobian matrix of the dynamical evolution relating the position and momentum variables at time $t$ with the corresponding at the origin of time). ${ }^{22}$ A periodic orbit is said to be stable if $|\operatorname{Tr} \mathbf{M}| \leqslant 2$, and unstable if $|\operatorname{Tr} \mathbf{M}|>2$. The trace of the stability matrix after $n$ iterations of the Poincare map can be related to the trace for one iteration as $^{23}$

$$
\operatorname{Tr}_{\mathbf{n}}=2 \cos \left[n\left\{\arccos \operatorname{Tr} \frac{\mathbf{M}_{1}}{2}\right\}\right]
$$

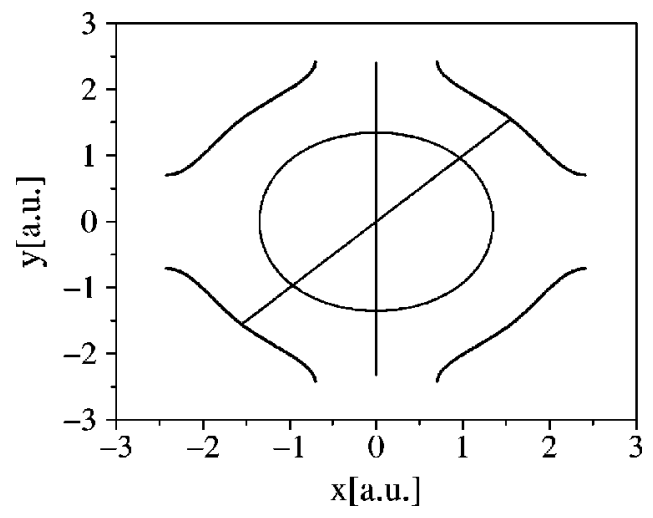

FIG. 2. The main period 1 periodic orbits of the system at $E$ $=80 \mathrm{meV}$, restricted to the Wigner-Seitz cell. The equipotential line at this energy is also shown.

for stable orbits, whereas for the unstable ones the cosine function is replaced by the hyperbolic cosine function. Because a new periodic orbit of period $n$ can appear (or one existing dissappear) only when $\operatorname{Tr} \mathbf{M}_{\mathrm{n}}=2$, from Eq. (13) we have that the possible bifurcations of period $n$ from a single period motion are given when

$$
\operatorname{Tr}_{1}=2 \cos \frac{2 \pi m}{n}
$$

with $m$ an integer number such that the argument of the cosine is modulo $\pi$. Unstable orbits do not bifurcate but they can change their stability. The possible types of bifurcations for two-dimensional Hamiltonian systems are only five $\mathrm{e}^{24}$ but, for our purposes, the most important one is the perioddoubling bifurcation $\left(\operatorname{Tr} \mathbf{M}_{1}=-2\right)$ which changes the stability of the period 1 motion.

Three principal periodic motions arise from the minima of the potential wells at hollow sites. They are depicted for illustration in Fig. 2 at $E=80 \mathrm{meV}$ (above the diffusion barrier) restricted to the Wigner-Seitz cell. One is an orbit parallel to the $x$ direction or, equivalently, the $y$ direction (due to the symmetry of the potential, all periodic orbits with a reflection symmetry by the plane $x=0$ have their counterpart in orbits with reflection symmetry by $y=0$ ). For energies below the saddle-point barrier, this orbit represents a frustrated parallel translation, but for $E>E_{s}$ it describes free drifting motions along the $x$ or $y$ directions. Because the frequencies of these two modes are degenerated, they can be added or substracted to give also two normal modes along the [100] or [010] azimuths (the diagonal directions). Note that the PES has also a reflection symmetry by the $x=y$ or $x=-y$ planes. This periodic orbit is localized for $E$ $<84.49 \mathrm{meV}$ and free above this energy. Finally, a circular type orbit, analogous to a frustrated rotation, also starts from the hollow minimum. This orbit describes a localized motion inside the potential well even at energies greater than $E_{\max }$, and it is responsible for the intrawell dynamics. We can understand the motion of the circular orbit at low energies as a combination of the frustrated translations along $x$ and $y$ with different phases, in analogy with the problem of normal 

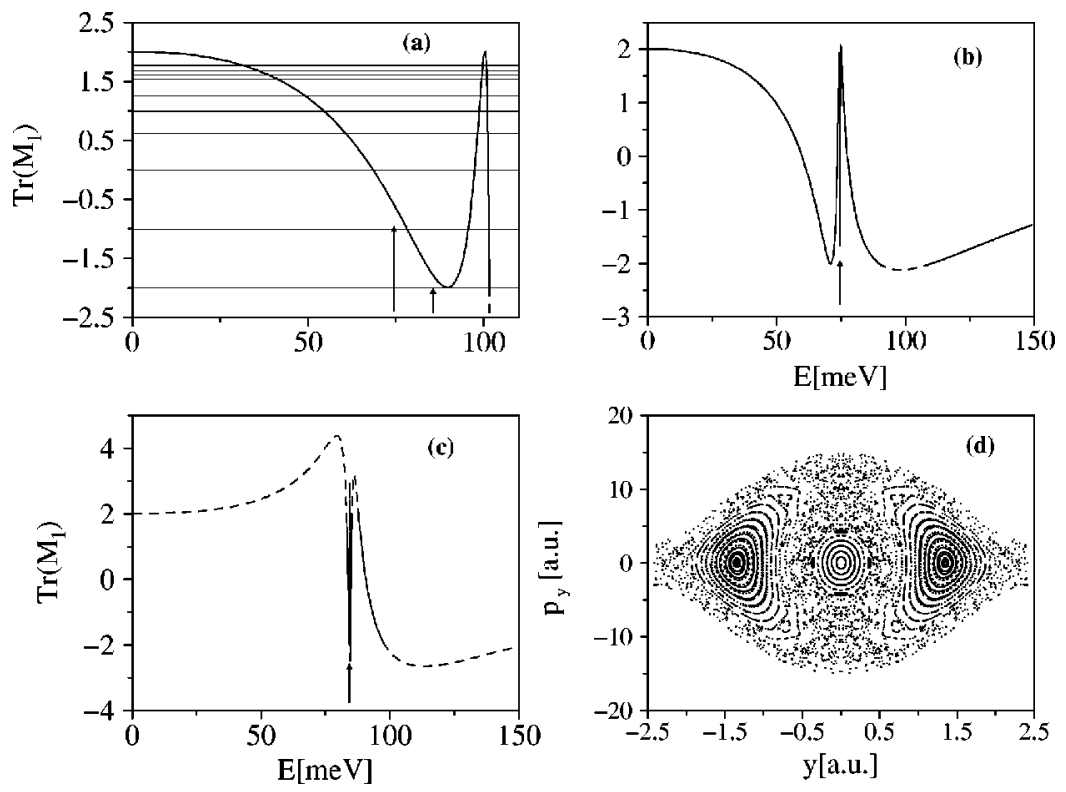

FIG. 3. Trace of the stability matrix of the principal motions as a function of the energy. (a) Circular orbit. A sequence of bifurcations (from period doubling at $\operatorname{Tr} \mathbf{M}_{1}=-2$ until period 15) is marked with solid thin lines. The arrows indicate the opening of energetic barriers $\left(E_{s}=74.6\right.$ and $E_{\max }=85.5$ ). (b) Parallel drifting motion. The energy $E_{s}$ is marked with an arrow. (c) Diagonal diffusive motion. The arrow points to the energy of the on top saddles at $84.49 \mathrm{meV}$. In all of them the stable orbits are plotted with solid lines and the unstable ones with dashed lines. (d) Poincare surface of section at $x=0, E=80 \mathrm{meV}$ showing the stability region originated by the stable circular orbit (left and right islands regions) and by the parallel diffusive motion (center islands chain). modes of vibration. ${ }^{25}$ The parallel and circular orbits are stable inside the well, but the diagonal one is unstable.

Close to the potential minima the system is nearly integrable, and information about the fundamental frequency of localized motions can be obtained by semiclassical quantization of the parallel translations. The Einstein-Brillouin-Keller quantization condition can be expressed as ${ }^{26}$

$$
\frac{1}{2 \pi} \oint_{C}\left(p_{x} d x+p_{y} d y\right)=\hbar\left(n+\frac{\mu}{4}\right), \quad n=0,1,2, \ldots,
$$

where the action integrals are calculated along topologically independent closed paths defining a torus in phase space, and $\mu$ is the Maslov index which depends only on the topology of the classical orbit $(\mu=2$ in our case). For the parallel translations, one of the momenta is always zero and the problem is reduced to one dimension, therefore the periodic orbit is semiclassically quantized by calculating its action integral along one period as we change the energy. When the quantization condition is matched for $n=0$, this will give an estimate of the fundamental frequency from $\omega_{0}=2 E / \hbar$. The value obtained is $\omega_{0}=6.4 \mathrm{meV}$ which agrees well with the experimentally determined frequency of the $T$ mode $^{5}$ (6 $\mathrm{meV})$. Note that by calculating energy differences at different values of $n$ in Eq. (15), we could also obtain an estimation of the anharmonicity by fitting to a first order anharmonic expansion the potential along the $x$ or $y$ directions. Although experimental measurements of anharmonicity have not been reported for this problem, such simple models have been shown to work well for adsorption of $\mathrm{CO}$ on a $\mathrm{Cu}(001)$ surface. ${ }^{27}$

For all of the three periodic motions, the trace of their stability matrices has been followed with the energy in order to detect their bifurcations and changes of stability which are reflected in Fig. 3. For the circular orbit, (a) panel, the opening of energetic barriers (indicated by arrows) does not seem to affect significantly its stability. Indeed, the orbit is quite stable (solid line) until $E \sim 101 \mathrm{meV}$ where it suddenly un- dergoes a series of bifurcations of decreasing periods. At $E$ $>101.6 \mathrm{meV}$, a period doubling bifurcation has turned this orbit very unstable (dashed line). No more stable localized motions inside a potential well are visible at higher energies. This is intuitively expected since localization is exclusively due to the effect of the potential wells and as we increase the energy the trajectories should follow more straight paths. However, it is interesting to see that about $15 \mathrm{meV}$ above $E_{\text {max }}$ localized motions are still stable.

For the straight motions along the $x, y$, or diagonal directions [(b) and (c) panels, respectively], the opening of energetic barriers change their character from localized to diffusing, although the topology of the orbit remains the same. For the motion along the [110] and [110] azimuths, the orbit is localized and stable until $E=E_{s}$, where it becomes a free drifting motion. This change in the PES originates an abrupt bifurcation [marked with an arrow in Fig. 3(b)] and the sudden onset of an infinite sequence of periodic orbits of all periods as the trace of the stability matrix approaches the value 2 [see Eq. (14)]. Diffusing orbits along the $x$ or $y$ directions with all possible periods are created. Because the main orbit remains stable an island around island structure is expected in the Poincaré surface of section [see Fig. 3(d) and Fig. 7]. This will originate a self-similar hierarchy of nested cantori which has been shown to be a possible mechanism for anomalous diffusion in Hamiltonian systems. ${ }^{12}$ After the transition region of abrupt bifurcation, the main orbit continues to be stable until $E=89.7 \mathrm{meV}$, where it suffers a period doubling bifurcation. Between this energy and $E$ $=110 \mathrm{meV}$ it remains unstable, and no stable drifting motions are seen to occur in the Poincare surface of section. Above $110 \mathrm{meV}$ it turns again to stable and remains so, as expected from the approaching to the integrable limit.

For the diagonal motion along the [100] azimuth [Fig. $3(\mathrm{c})$ ] a similar situation is expected, the difference being that due to the nonseparable term of the potential $V_{2}(x, y)$ around the minima, the motion is highly nonlinear in the diagonal direction and the orbit is unstable. The interesting situation is 
that, when the energetic barrier is open for this orbit to be diffusing (at $E=84.5 \mathrm{meV}$ ), it coalesces with an orbit of the same topology originated at the small minima of the on-top sites and which in this case is stable. The abrupt bifurcation originating drifting diagonal motions of all periods is seen again, and the main diagonal orbit is stabilized by the minima so that for $87.8 \mathrm{meV} \leqslant E \leqslant 97.5 \mathrm{meV}$ it is stable, and then becomes unstable until, at $E>150 \mathrm{meV}$, it starts approaching the integrable limit. The stabilization of this diagonal motion will originate long jumps along the [100] and [010] azimuths, and this is consistent with the experimental observation that a high rate of diffusing paths along this direction also exists in addition to the paths across the saddle points along [110] or [110] azimuths.

We note that inside the small potential wells at the on-top sites the same three periodic motions described above are present, but now the parallel frustrated modes are unstable and the diagonal ones are stable. The rotational frustrated motion is also stable only for energies below the on-top maxima (between 82.74 and $85.51 \mathrm{meV}$ ). When this barrier is opened the motion turns unstable, opposite to what happened in the potential wells at the hollow sites. By semiclassically quantizing the (stable) diagonal periodic motion, we obtain a fundamental frequency $\omega_{0}^{\text {top }} \sim 2.4 \mathrm{meV}$. Although for the present system the experimental results cannot conclude about the existence of on-top site adsorption, it is intriguing to see that a weaker mode at approximately half the frequency of the $T$ mode has been reported. ${ }^{4}$ Indeed, there have been observations of stable on-top bound states for potassium on $\mathrm{Cu}(111)$ and $\mathrm{Ni}(111){ }^{28}$

\section{B. Power spectra and anomalous diffusion}

In this section we will analyze velocity power spectra at several energies discriminated by the different dynamical behaviors discussed in the preceding section. Our finding is that, for the small range of energies between the barrier saddle and the top $(74.6 \leqslant E \leqslant 85.5)$, anomalous diffusion dominates due to a trapping mechanism of trajectories around islands of stability due to long drifting motions. After a transition region around $90 \mathrm{meV}$ in which the transport properties cannot be unambiguously assigned as normal or enhanced diffusion, normal diffusion takes place due to the increasing chaoticity of the phase space and remains so until $E \sim 125 \mathrm{meV}$. Then we have weakly enhanced diffusion while approaching the integrable limit where the ballistic diffusion regime readily settles (close to $200 \mathrm{meV}$ ). One of the aims of the present section is also to relate the anomalous diffusion process to Lévy flights, testing some statistical models proposed to analyze chaotic systems. The use of Lévy distributions has also consequences for the $\Delta \mathbf{K}$ dependence of the dynamic structure factor.

At $75<E \leqslant 89.5 \mathrm{meV}$, both the circular bounded orbit and the open parallel motion along the $x$ and $y$ directions are stable. Due to this fact, the typical picture of a chaotic trajectory at these energies will be that of a random walker performing long free paths along $x$ or $y$, interrupted by more or less long periods of localization inside a unit cell. A trajectory at $84 \mathrm{meV}$ is shown in Fig. 4. Such random walks

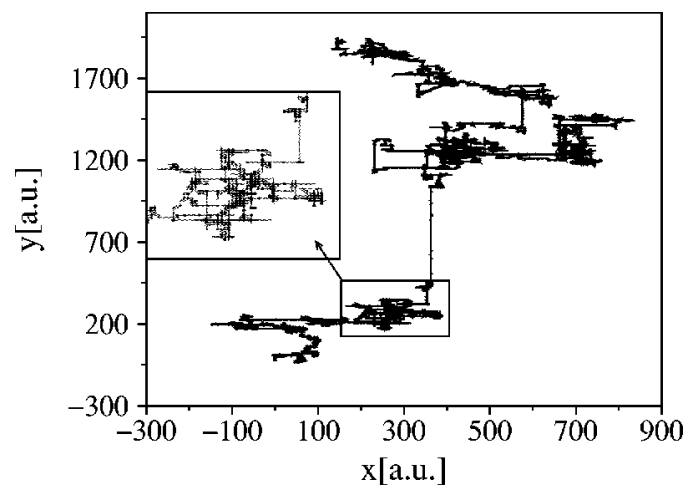

FIG. 4. A chaotic trajectory at $E=84 \mathrm{meV}$, run until $t$ $\sim 19000$ ps. Shown in the inset there is a magnification of the rectangular box.

have been analytically described by several statistical models and shown to lead to Lévy type distributions and anomalous transport. ${ }^{29,30}$ To see that this is also the situation in our system, we have propagated until long times ensembles of chaotic trajectories (chosen randomly inside the stochastic regions of the phase space) at different energies. Several relevant correlation functions were calculated, in particular, the velocity autocorrelation function and the MSD, both directly related to the diffusion constant. In Fig. 5, we present the velocity power spectra as obtained from Eq. (5) at two different energies inside this regime, (a) $E=84 \mathrm{meV}$ and (b) $E=80 \mathrm{meV}$. At small frequencies (the frequencies have been rescaled by the harmonic frequency of the potential well $\omega_{0}=2 \pi \sqrt{V_{0} / m a^{2}}$ ), we observe the predicted behavior
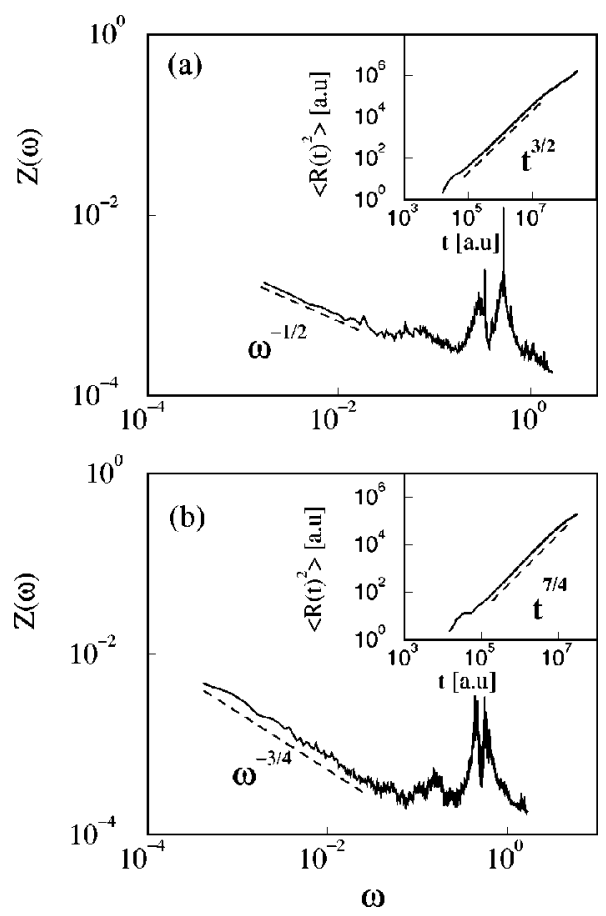

FIG. 5. Velocity power spectra and MSD (in the insets), at two different energies: (a) $E=84 \mathrm{meV}$ and (b) $E=80 \mathrm{meV}$. The frequencies are given in units of the harmonic frequency $\omega_{0}$ $=2 \pi \sqrt{V_{0} / m a^{2}}$. 
for anomalous diffusion $Z(\omega) \sim \omega^{-\alpha}$ with $\alpha \sim 1 / 2$ and $\alpha \sim 3 / 4$ for 84 and $80 \mathrm{meV}$, respectively. The MSD and the velocity autocorrelation function are related at long times by

$$
\left\langle\mathbf{R}^{2}(t)\right\rangle \sim 2 t \int_{0}^{t}\langle\mathbf{v}(0) \cdot \mathbf{v}(\tau)\rangle d \tau .
$$

Therefore for a divergence of the power spectrum at small $\omega$ as $\omega^{-\alpha}$, the MSD should diverge at long times as $\left\langle\mathbf{R}^{2}(t)\right\rangle$ $\sim t^{1+\alpha}, \quad 0<\alpha<1$, or $\left\langle\mathbf{R}^{2}(t)\right\rangle \sim t^{2}$ (ballistic diffusion) for $\alpha \geqslant 1$. The numerically calculated MSD are shown in the insets of Fig. 5, together with the predicted values of $t^{1+\alpha}$ form, demonstrating the consistency of the numerical values for the slope of the power spectra discussed above. Basically two models have been employed in a theoretical description of anomalous diffusion based on Lévy statistics. The theory by Geisel et al. ${ }^{12,13,29}$ uses a renewal formalism, and assumes that a random walker performs statistically independent free paths of constant velocity $v_{0}$ whose durations $t$ (or lengths $l$ ) are distributed according to an inverse power law

$$
\phi(l) \sim l^{-\beta} .
$$

Other authors have employed a continuous time random walk formalism ${ }^{14,30}$ in which particles move at constant velocity for a given time, then stop and choose a new direction and time of sojourn at random according to given probabilities. The central quantity is $\psi(l, t)$, the probability density to move a distance $l$ at time $t$ in a single motion event. This quantity can be expressed as

$$
\psi(l, t)=p(l \mid t) \phi(l),
$$

where $p(l \mid t)$ is the conditional probability for a transition at time $t$, given that the displacement is $l$, and $\phi(l)$ is the jump length probability. When assuming an inverse power law for $\phi(l)$ as in Eq. (17), and $p(l \mid t)=\delta\left(t-|l| / v_{0}\right)$ both theories give the same result ${ }^{29}$ for the behavior of the MSD and therefore the power spectra. In particular, for the MSD the predicted asymptotic behavior is ${ }^{12,29,30}$

$$
\left\langle\mathbf{R}^{2}(t)\right\rangle \sim \begin{cases}t^{2} & 1<\beta \leqslant 2, \\ t^{4-\beta} & 2<\beta<3, \\ t \ln t & \beta=3, \\ t & \beta>3 .\end{cases}
$$

In order to verify the validity of the statistical models in our system, we have calculated the jump length probabilities $\phi(l)$ at energies where anomalous diffusion was observed. At these energies, the potential barriers constraint the diffusive motion to the $x$ or $y$ directions, and we can define the beginning of a jump in $x$ or $y$ when a trajectory crosses the activation barrier along one of these directions, finishing the jump when the momentum in this direction is reversed. Between two jumps generally there is a waiting time inside a given unit cell where the trajectory executes chaotic motion and looses memory of the initial conditions, therefore two consecutive jumps are statistically independent. This picture is consistent with the models discussed above. Note that, due to the symmetry of the PES, the distribution of jumps in $x$ or

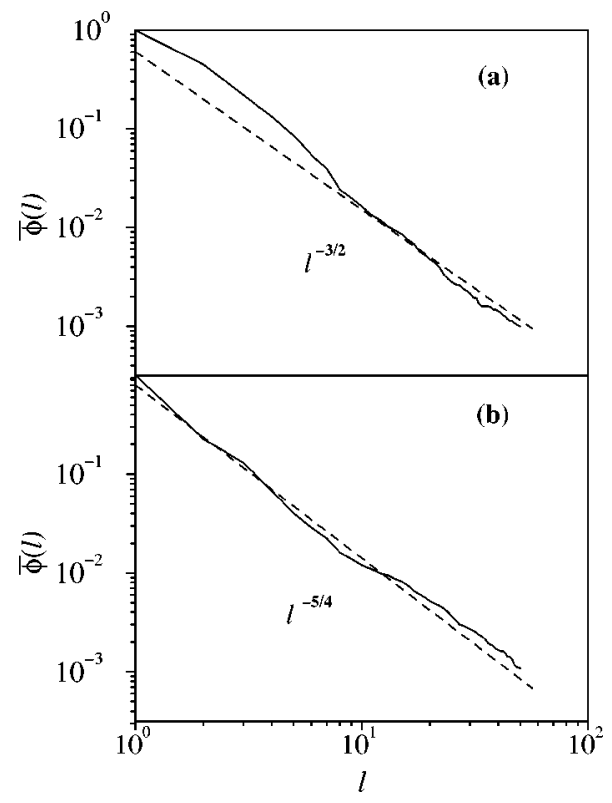

FIG. 6. Integrated jump probability distributions (solid lines) at (a) $E=84 \mathrm{meV}$ and (b) $E=80 \mathrm{meV}$. Only jumps with lengths less than 50 unit cells are shown. The theoretical predictions given by Eq. (19) are plotted with dashed lines.

$y$ is identical. At higher energies, the particle can move freely along any direction and the criterium for the termination of a free jump is not so clear. We have found a more convenient way to count jumps by calculating the Gaussian curvature at every integration step of a trajectory defined as

$$
\kappa=\frac{|\dot{x} \ddot{y}-\ddot{x} \dot{y}|}{\left(\dot{x}^{2}+\dot{y}^{2}\right)^{3 / 2}}
$$

and the end (beginning) of a jump is considered to be the point of the trajectory where the radius of curvature $\rho$ $=\kappa^{-1}$ is less than some critical value $\rho_{c}$. This value is somewhat arbitrary and we chose it empirically at every energy to agree with our intuitive notion of a flight. The curvature criterium was proposed by Sholl and Skodje in his investigation of Lévy flights in the Xe/Pt diffusion system. ${ }^{31}$ At energies below the top of the hill, for instance, we have found identical jump distributions $\phi(l)$ by using the first criterium of momentum change and the curvature criterium choosing $\rho_{c}=0.15 a_{0}$.

To have a good statistics it is better to calculate the integrated jump distribution as

$$
\bar{\phi}(l)=\int_{l}^{\infty} \phi\left(l^{\prime}\right) d l^{\prime}
$$

and the corresponding $\phi(l)$ is obtained by differentiation of $\bar{\phi}(l)$. In Fig. 6 we present the integrated jump length probabilities (using as discrete jump length $l$ the number of unit cells traversed in the jump) at the two energies of the power spectra of Fig. 5. The theoretical predictions of the statistical models should give the behavior $\bar{\phi}(l) \sim l^{-3 / 2}$ and $\bar{\phi}(l)$ $\sim l^{-5 / 4}$ for 84 and $80 \mathrm{meV}$, respectively, shown in the figure with a dashed line. The good agreement confirms the validity 


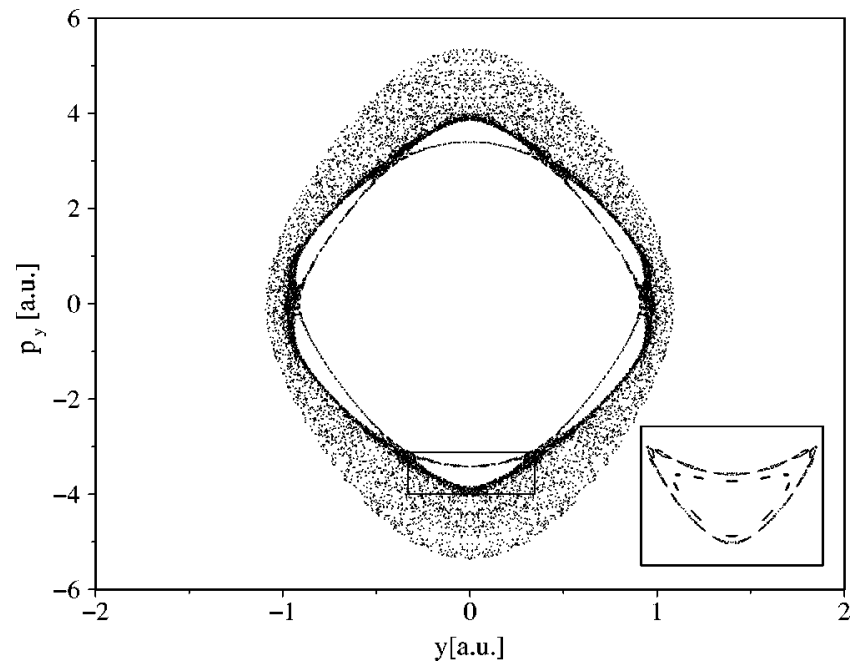

FIG. 7. Poincaré surface of section in $x=a / 2$ at $E=84 \mathrm{meV}$ illustrating the islands around islands structure of the main parallel diffusing orbit. The inset shows some islands chains magnified from the squared region.

of this Lévy flight model for our system. The reason for anomalous diffusion lies on the island around island structure in phase space originated by the abrupt bifurcation of the parallel drifting motion (see Fig. 7). This causes sticking of the chaotic trajectories around cantori giving diffusive motion of all possible jump lengths.

The existence of stable or Lévy distributions of the type (17) affects the $\Delta \mathbf{K}$ behavior of the dynamic structure factor. To see this, suppose a one-dimensional random walker with the algebraic jump length probability distribution (17) for the individual steps (a Weierstrass random walker ${ }^{11}$ ). Then, the probability density for the walker to be at position $x$ after $N$ steps is the convolution

$$
G_{N}(x)=\phi(l) \times \cdots \times \phi(l)
$$

$N$ times and where, by definition, $G_{N}(x)$ is a discrete time one-dimensional pair correlation function. In Fourier space, the intermediate scattering function can then be expressed as

$$
I_{N}(\Delta K)=\hat{\phi}(\Delta K)^{N} .
$$

For the probability distribution (17), the characteristic function $\hat{\phi}(\Delta K)$ has the limiting $\Delta K \rightarrow 0$ form $^{11}$

$$
\hat{\phi}(\Delta K)=e^{-c(\beta)|\Delta K a|^{\beta-1}},
$$

where $a$ is the unit cell length. By defining the time $t$ $=N \Delta t$ and a generalized diffusion coefficient as $\mathcal{D}$ $=c(\beta) \lim _{a, \Delta t \rightarrow 0}\left(a^{\beta-1} / \Delta t\right)$, and using Eq. (3), one readily finds for the dynamic structure factor at small $\Delta K$ the functional form

$$
S_{s}(\Delta K, \omega)=\frac{1}{\pi} \frac{\mathcal{D} \Delta K^{\beta-1}}{\omega^{2}+\mathcal{D}^{2} \Delta K^{2(\beta-1)}} .
$$
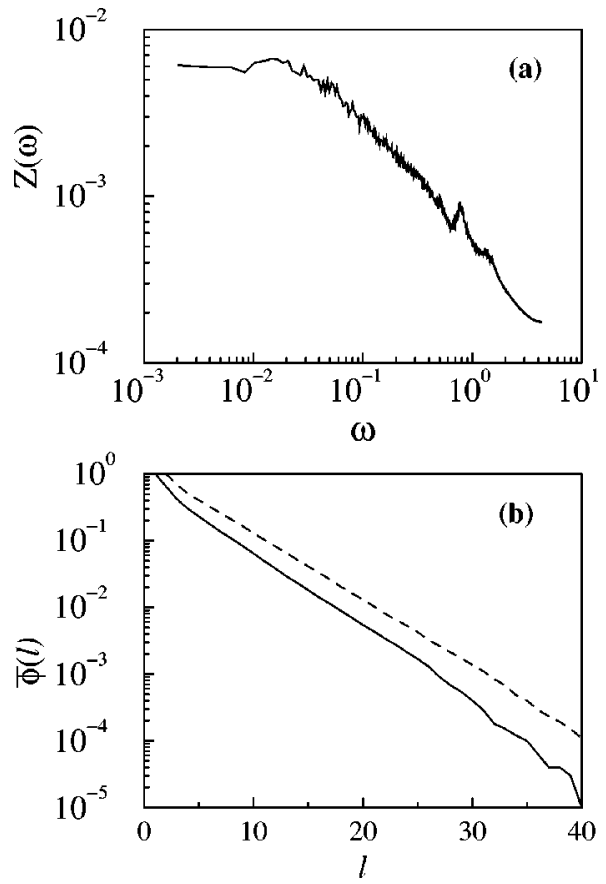

FIG. 8. (a) Velocity power spectrum at $E=95 \mathrm{meV}$, with frequencies in units of $\omega_{0}$. (b) Solid line: integrated jump length probability at the same energy. Dashed line: Integrated probability distribution for jump duration times. Both give an exponential decay and the slopes coincide within statistical uncertainty.

Note that within this Weierstrass random walk approach, when $\beta=3$ the characteristic function (24) is a Gaussian and we recover the result for normal diffusion, Eq. (4).

For energies above $90 \mathrm{meV}$ (until $\sim 120 \mathrm{meV}$, still far away from the integrable limit) we have observed only normal diffusion. The MSD's grow linearly in time and the power spectra converge to a finite value as $\omega \rightarrow 0$ [see Fig. $8(a)$. By using the curvature criterium, we have found an exponential behavior of the jump length probabilities, $\phi(l)$ $\sim e^{-\gamma l}$. The corresponding integrated probability is shown in Fig. 8(b), together with the sojourn time distribution. The agreement between the length and duration time probabilities is an additional argument in favor of the statistical models used, since both of them assume that during a jump the velocity is constant.

In spite of the absence of anomalous diffusion, at several of these energies we still have stable diffusive motions which originate long jumps. For instance, when $88 \mathrm{meV} \leqslant E$ $\leqslant 97.5 \mathrm{meV}$ the diagonal orbit is stable, and when $E$ $>110 \mathrm{meV}$ the parallel orbit becomes stabilized again. The influence of this change of stability can be traced by relating the microcanonical diffusion coefficient to average jump lengths using some approximate statistical theories for the diffusion coefficient. For instance, for extremely mobile adatoms we can use the kinetic theory of gases ${ }^{32}$ to express the diffusion coefficient as

$$
D(E)=\frac{1}{2}\langle v\rangle \lambda
$$




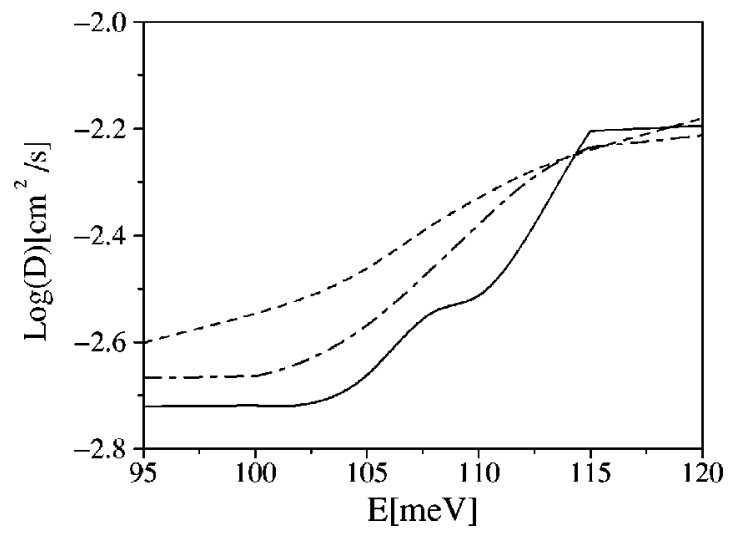

FIG. 9. Microcanonical diffusion coefficients for an energy range between 95 and $120 \mathrm{meV}$. Solid line: exact diffusion coefficient from a fitting to the Einstein relations using microcanonical ensembles. Dashed line: Approximation from the kinetic theory of gases Eq. (26). Dot-dashed line: Random flight approximation Eq. (27).

where $\langle v\rangle$ is the average velocity and $\lambda$ is the mean free path. Correlations due to long flights are manifested as anomalous high values for $\lambda$. Another approximate expression for the microcanonical diffusion coefficient comes from the generalization of the simple random walk picture to a random flight formalism ${ }^{31,33}$ which in our case gives

$$
D(E)=\frac{1}{2} \nu\left\langle l^{2}\right\rangle,
$$

where $\nu$ is the frequency of flights and $\left\langle l^{2}\right\rangle$ the average squared length of the jumps. The frequency $\nu$ can be computed as the total number of flights containing a nonzero number of barrier crossings, divided by the total time. This model assumes that successive flights are uncorrelated. If the jumps consist of only single hops, one obviously should have $\left\langle l^{2}\right\rangle=a^{2}$. In Fig. 9 we plot the exact microcanonical diffusion coefficient obtained from molecular dynamics simulations using the Einstein formula $D(E) \sim\left\langle\mathbf{R}^{2}(t)\right\rangle / 4 t$ [or analogously Eq. (6)], versus the two approximations, Eqs. (26) and (27). It is seen that around $E=110 \mathrm{meV}$ the true diffusion coefficient increases abruptly, due to the stabilization of the main parallel diffusing orbit. Although the two approximations used overestimate the diffusion coefficient at low energies, it is observed that the same qualitative feature is present in both models, that is, a more or less pronounced increase of the diffusion constant around $110 \mathrm{meV}$. In both approximations this is due to the increase in the jump length caused by the diffusing periodic orbit.

\section{DISCUSSION AND CONCLUSIONS}

We have investigated in detail the deterministic chaotic dynamics and transport properties of $\mathrm{Na}$ adparticles on a $\mathrm{Cu}(001)$ surface using a semiempirical potential..$^{5}$ This system has received considerable attention recently as a paradigm to study atomic diffusion by the QHAS experimental technique. A question of interest is the role played by the PES in the diffusion mechanism. This question arised be- cause using this technique, one can estimate the rates of migration paths along different directions as well as the contribution of long jumps to the diffusion coefficient. Although a quantitative study requires the inclusion of some kind of frictional coupling and thermal effects, as was done in Ref. 5, the deterministic dynamics can explain well some qualitative features of the experiment.

It has been found that for a range of energies from the activation barrier $(\sim 74.6 \mathrm{meV})$ until well above the top barrier, the phase space shows a coexistence of chaotic regions with stability islands produced by the simplest periodic motions. The periodic motions are of two kinds: those producing localization inside a potential well, and therefore inhibiting diffusion, and those promoting long jumps along the $x$, $y$ directions as well as the diagonal ones. Inside a potential well we have found stable localized motions associated to fundamental frequencies of vibration. By semiclassical quantization of these motions, we have obtained an estimation of the frustrated translational mode ( $T$ mode) in good agreement with the experimentally measured value. It is remarkable that localized motions are stable for energies much higher than the saddle point barrier. The orbits corresponding to frustrated parallel translations suffer an abrupt bifurcation when energy barriers are opened, and originate an islands around islands structure in the phase space consisting of drifting motions of all possible periods. The diagonal translations are unstable but experience the same sudden bifurcation with energy. The interesting situation is that this diagonal orbit becomes stabilized by the small minima at the ontop sites, and we suggest this stability as a possible explanation for the unusual high rate of migration paths along this direction detected in the experiment.

Our second aim has been to investigate the possibility of anomalous transport mechanisms in this potential, and if this is the case to provide an explanation for this mechanism in terms of simple periodic motions. For energies between the diffusion barrier and slightly above the potential maximum, both the localized and parallel diffusing motions are stable, and particles behave as a random walker executing free jumps of constant velocity in random directions after some waiting time in an equilibrium position. Such statistical models have been investigated in the past in connection to chaotic Hamiltonian systems and Lévy flights. ${ }^{9,14}$ Here we have found that anomalous diffusion is present in our system for the range of energies where both stable motions coexist, and that the theoretical predictions of the model are able to reproduce satisfactorily the power spectra and MSD's numerically calculated. At higher energies (from 95 up to $\sim 120$ $\mathrm{meV}$ ), we have seen only normal diffusion. However, long jumps still play an important role in the deterministic diffusion mechanism, since at several of these energies diffusive motions become stabilized. We have calculated microcanonical diffusion coefficients in this energy range using molecular dynamics simulations and two approximate statistical expressions, and verified the influence of long jumps due to the stabilization of the free diffusing motion. The influence of long correlated flights has also been investigated theoretically in other atom-surface diffusing systems with experimental results, ${ }^{31}$ and although long jumps were determined 
to be important, deterministic anomalous diffusion was not observed.

An important question to be addressed is whether a full consideration of the diffusion process, taking into account the friction, the coupling to the perpendicular motion and the surface temperature through random forces will alter the dynamics to the extent of washing out any deterministic effect. When introducing dissipation, the stable periodic orbits become sinks or attracting centers ${ }^{7}$ and the invariant tori (islands) are destroyed. Both regular and chaotic motions become transient since eventually all trajectories settle down in one of the sinks (minima at the hollow or the on-top sites). However, the existence of an external force, even if it is random, will eventually provide enough energy to escape an attractor and give rise to diffusive motion. The assumption that the system is in thermal equilibrium and that the dissipation mechanism is of the form $-\eta \dot{\mathbf{R}}$ defines the statistical properties of the noise through the fluctuation-dissipation theorem, and these are those of a Gaussian white noise. ${ }^{15,34}$ Therefore, modelling the dynamics by a Langevin equation of the type (2), as has been done to simulate the experiments, ${ }^{5}$ is expected to show only normal diffusion in the long time run (one can also verify that the Maxwell-Boltzmann distribution is a stationary solution of the corresponding Klein-Kramers equation; ${ }^{15}$ furthermore this stationary solution is unique). However, the thermal equilibration time needed for the stationary behavior should be larger than $\eta^{-1}$. If the friction constant is small enough, it is possible that the thermal equilibration time is much bigger than the equilibration time due to the chaotic dynamics, and under such circumstances one could observe a transient region in the MSD or the power spectrum where the adiabatic or systematic potential plays an important role. The intensity of the noise (temperature) should be also small so that the deterministic dynamics is not strongly perturbed.

In Fig. 10(a) we have plotted a Poincaré surface of section of the full Langevin dynamics, Eq. (2), at a value of the friction coefficient $\eta=10^{-3} \mathrm{ps}^{-1}$ and $T=50 \mathrm{~K}$ [compare to Fig. 3(d)]. We have numerically solved this equation using a velocity Verlet method ${ }^{35}$ and an accurate fourth order stochastic Runge-Kutta method ${ }^{36}$ since both are adequate for treating properly the dynamics, especially at low values of friction. It is seen that the island structure coming from the main parallel diffusing motion around $y=p_{y}=0$ becomes blurred, but still there are many orbits giving narrow bands around this center, therefore resembling quasiperiodic motion of the free diffusive type.

The corresponding velocity power spectrum and MSD for a canonical ensemble with an initial Maxwell distribution for the velocities is shown in Fig. 10(b). Note that thermal equilibration times are of the order of $10^{3} \mathrm{ps}$, while dynamical equilibrium is reached at about 100 ps. This can be appreciated in the inset, where we can distinguish two different slopes for the MSD, one giving "transient" anomalous diffusion and other normal diffusion, and the same can be seen in the behavior of the power spectrum at small frequencies.
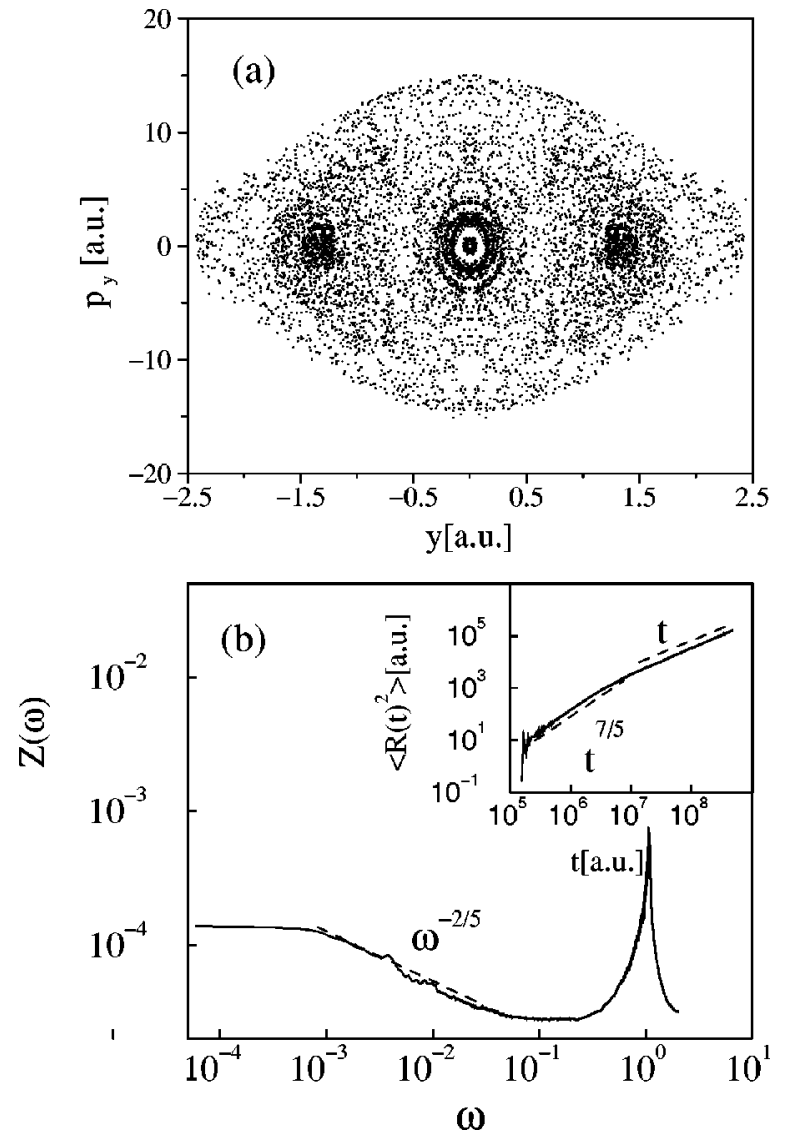

FIG. 10. (a) Poincaré surface of section at $x=0$ of the full Langevin dynamics, Eq. (2), at $T=50 \mathrm{~K}$ and $\eta=10^{-3} \mathrm{ps}^{-1}$. (b) Velocity power spectrum and MSD (inset) for an ensemble of 1000 trajectories with a Maxwell distribution of velocities at the same temperature. Frequencies in units of the fundamental frequency $\omega_{0}$.

At finite frequencies a broad band around $\omega=\omega_{0}$ (recall that the frequencies are scaled by $\omega_{0}$ ) appears showing the fingerprint of the frustrated translational mode. Since experimental time scales are finite, this transient anomalous diffusion could be observed in some systems (for this paricular one, $\eta$ was found to be around $1 \mathrm{ps}^{-1}$ (Ref. 1) and transient anomalous diffusion can not be seen). It is worth noting that for diffusion of $\mathrm{Xe}$ atoms on a $\mathrm{Pt}(111)$ surface the extreme case of ideal gas behavior (zero effective friction and no influence of the adiabatic potential, corresponding to ballistic diffusion) has been observed experimentally. ${ }^{37}$ We also mention that anomalous diffusion has been described in the context of the Langevin formulation in nonequilibrium systems, for instance by introducing correlated (colored) noise. ${ }^{38}$

\section{ACKNOWLEDGMENTS}

This work has been supported by DGES (Spain) under Contracts No. PB95-0071 and the European Contract No. HPRN-CT-1999-00005. R. Guantes acknowledges a grant from Comunidad Autónoma de Madrid, Spain. 
${ }^{1}$ J.W.M. Frenken and B.J. Hinch, in Helium Atom Scattering from Surfaces, edited by E. Hulpke, Springer Series in Surface Sciences Vol. 27 (Springer-Verlag, New York, 1992), p. 287.

${ }^{2}$ L. van Hove, Phys. Rev. 95, 249 (1954).

${ }^{3}$ J.P. Hansen and I.R. McDonald, Theory of Simple Liquids (Academic Press, London, 1986).

${ }^{4}$ J.P. Ellis and J.P. Toennies, Phys. Rev. Lett. 70, 2118 (1993); L.Y. Chen and S.C. Ying, ibid. 71, 4361 (1993).

${ }^{5}$ A.P. Graham, F. Hofmann, J.P. Toennies, L.Y. Chen, and S.C. Ying, Phys. Rev. Lett. 78, 3900 (1997); Phys. Rev. B 56, 10567 (1997).

${ }^{6}$ L.Y. Chen and S.C. Ying, Phys. Rev. B 49, 13838 (1994).

${ }^{7}$ A.J. Lichtenberg and M.A. Lieberman, Regular and Chaotic Dynamics (Springer-Verlag, New York, 1992).

${ }^{8}$ P. Gaspard, Chaos, Scattering and Statistical Mechanics, Nonlinear Science Series IX (Cambridge University Press, Cambridge, 1998).

${ }^{9}$ T. Geisel, in Lévy Flights and Related Topics in Physics, edited by M. F. Schlesinger, G. M. Zaslavsky, and U. Frisch (SpringerVerlag, Berlin, 1995).

${ }^{10}$ M.F. Schlesinger, G.M. Zaslavsky, and J. Klafter, Nature (London) 363, 31 (1993).

${ }^{11}$ W. Paul and J. Baschnagel, Stochastic Processes (SpringerVerlag, Berlin, 1999).

${ }^{12}$ T. Geisel, A. Zacherl, and G. Radons, Phys. Rev. Lett. 59, 2503 (1987); Z. Phys. B 71, 117 (1988).

${ }^{13}$ T. Geisel, J. Wagenhuber, P. Niebauer, and G. Obermair, Phys. Rev. Lett. 64, 1581 (1990); Phys. Rev. B 45, 4372 (1992).

${ }^{14}$ J. Klafter and G. Zumofen, Phys. Rev. E 49, 4873 (1994).

${ }^{15}$ H. Risken, The Fokker-Planck Equation (Springer-Verlag, Berlin, 1984).

${ }^{16}$ R. Ferrando, R. Spadacini, and G.E. Tommei, Phys. Rev. E 48, 2437 (1993).

${ }^{17}$ C.T. Chudley and R.J. Elliott, Proc. Phys. Soc. London 77, 353 (1961).

${ }^{18}$ S.-A. Lindgren, C. Svensson, and L. Wallden, Phys. Rev. B 42, 1467 (1990).
${ }^{19}$ L.Y. Chen, M.R. Baldan, and S.C. Ying, Phys. Rev. B 54, 8856 (1996).

${ }^{20}$ R. Guantes, F. Borondo, and S. Miret-Artés, Phys. Rev. E 56, 378 (1997).

${ }^{21}$ A. Weinstein, Invent. Math. 20, 47 (1973).

${ }^{22}$ R. Seydel, Practical Bifurcation and Stability Analysis (SpringerVerlag, New York, 1994).

${ }^{23}$ J.-M. Mao and J.B. Delos, Phys. Rev. A 45, 1746 (1992).

${ }^{24}$ K.R. Meyer, Trans. Am. Math. Soc. 149, 95 (1970); M.A.M. de Aguiar, C.P. Malta, M. Baranger, and K.T.R. Davies, Ann. Phys. (N.Y.) 180, 167 (1987).

${ }^{25}$ E.B. Wilson, Jr., J.C. Decius, and P.C. Cross, Molecular Vibrations (Dover, New York, 1980), Chap. 2.

${ }^{26}$ I.C. Percival, Adv. Chem. Phys. 36, 1 (1977).

${ }^{27}$ A. Graham, F. Hofmann, and J.P. Toennies, J. Chem. Phys. 104, 5311 (1996).

${ }^{28}$ D.L. Adler, I.R. Collins, X. Liang, S.J. Murray, G.S. Leatherman, K.-D. Tsuei, E.E. Chaban, S. Chandavarkar, R. McGrath, R.D. Diehl, and P.H. Citrin, Phys. Rev. B 48, 17445 (1993).

${ }^{29}$ T. Geisel, J. Niertwetberg, and A. Zacherl, Phys. Rev. Lett. 54, 616 (1985); M.F. Shlesinger and J. Klafter, ibid. 54, 2551 (1985).

${ }^{30}$ G. Zumofen and J. Klafter, Phys. Rev. E 47, 851 (1993); J. Klafter, M.F. Shlesinger, and G. Zumofen, Phys. Today 49, 33 (1996).

${ }^{31}$ D.S. Sholl and R.T. Skodje, Physica D 71, 168 (1994).

${ }^{32}$ S.J. Lombardo and A.T. Bell, Surf. Sci. Rep. 13, 1 (1991).

${ }^{33}$ S. Chandrasekhar, Rev. Mod. Phys. 15, 1 (1943).

${ }^{34}$ P. Reimann, Chem. Phys. 268, 337 (2001).

${ }^{35}$ M.P. Allen and D.J. Tildesley, Computer Simulations of Liquids (Clarendon Press, Oxford, 1987).

${ }^{36}$ E. Hershkovitz, J. Chem. Phys. 108, 9253 (1998); see also R.L. Honeycutt, Phys. Rev. A 45, 600 (1992).

${ }^{37}$ J. Ellis, A.P. Graham, and J.P. Toennies, Phys. Rev. Lett. 82, 5072 (1999).

${ }^{38}$ S.I. Denisov and W. Horsthemke, Phys. Rev. E 62, 7729 (2000), and references therein. 